\title{
HispanismeS
}

Revue de la Société des Hispanistes Français

$18 \mid 2021$

Murs, barrières, obstacles dans les mondes

hispaniques II

\section{Poétique des murs traversés entre Málaga et Larache dans Sombras en sepia de Sergio Barce Gallardo, 2006}

Poetics of the Walls crossed between Málaga and Larache in Sombras en sepia by Sergio Barce Gallardo, 2006

Poética de los muros cruzados entre Málaga y Larache en Sombras en sepia de Sergio Barce Gallardo, 2006

\section{Lydie Royer}

\section{(2penEdition}

\section{Journals}

Édition électronique

URL : https://journals.openedition.org/hispanismes/14007

DOI : $10.4000 /$ hispanismes. 14007

ISSN : 2270-0765

\section{Éditeur}

Société des Hispanistes Français

\section{Référence électronique}

Lydie Royer, « Poétique des murs traversés entre Málaga et Larache dans Sombras en sepia de Sergio Barce Gallardo, 2006 », HispanismeS [En ligne], 18 | 2021, mis en ligne le 31 décembre 2021, consulté le 30 janvier 2022. URL : http://journals.openedition.org/hispanismes/14007 ; DOI : https://doi.org/ 10.4000/hispanismes.14007

Ce document a été généré automatiquement le 30 janvier 2022.

\section{cc) $(\mathrm{S} \odot$}

Les contenus de cette revue sont mis à disposition selon les termes de la Licence Creative Commons Attribution - Pas d'Utilisation Commerciale - Pas de Modification 4.0 International. 


\section{Poétique des murs traversés entre} Málaga et Larache dans Sombras en sepia de Sergio Barce Gallardo, 2006

Poetics of the Walls crossed between Málaga and Larache in Sombras en sepia by Sergio Barce Gallardo, 2006

Poética de los muros cruzados entre Málaga y Larache en Sombras en sepia de Sergio Barce Gallardo, 2006

\section{Lydie Royer}

\section{Introduction}

«L'un à l'autre est ce qu'est la couleur à l'ombre,

l'or du fruit mûr à l'or de la feuille sèche. » Yves Bonnefoy (1923-2016)

Le roman espagnol Sombras en sepia ${ }^{1}$, publié par Sergio Barce Gallardo en 2006 raconte l'aventure d'un homme Abel Egea qui découvre Nadja, une jeune Marocaine de dix-sept ans et son fils arrivés clandestinement sur une plage de Cádiz. Une rencontre bouleversante qui se fait derrière un petit mur, mot récurrent aux connotations doubles, associant, dans ce roman, présent et passé, réel et imaginaire, désir et frustration, dehors et dedans, corps et âme, lumière et ombre. Autant de facettes du mur que nous analyserons d'abord dans le déplacement du personnage principal, puis à travers les chronotopes ${ }^{2}$ et les discours qui interrogent, dans sa complexité, la construction imaginaire et politique de la barrière entre l'Espagne et le Maroc. Nous étudierons ensuite le mur dans sa dimension métaphorique en nous appuyant sur l'opacité définie dans la Poétique d'Édouard Glissant comme les profondeurs ${ }^{3}$ que l'homme porte en lui ou les ombres épaisses qui s'opposent au passage d'une chose. 


\section{La traversée du Détroit de Gibraltar}

2 Le protagoniste, Abel Egea, un veuf espagnol, retraité, est le double de l'écrivain qui avait émigré au Maroc avec sa famille, au début du xxe siècle avant de retourner en Espagne à la fin du Franquisme. Les deux mots du titre illustrent donc le cheminement de l'écrivain en quête des ombres de son passé. Le roman s'ouvre sur le mouvement lent du personnage, envahi par la solitude, et qui marche sur une plage andalouse, attiré par un mur. Les caractéristiques du mur "petit et agonisant » qu'il observe de loin, préfigurent la scène de détresse qu'il découvre derrière les décombres d'une buvette calcinée :

Abel Egea se acercó y miró detrás del muro agonizante, un alambre oxidado le rozó la mano. Justo detrás de ese pequeño muro, una chica desharrapada, sostenía en el regazo un bebé dormido. Luego recordaría que no fue ese niño que le llamó la atención sino los ojos almendrados [...].Unos ojos de cervatillo, agitados y temerosos [...] que le hicieron dar un paso atrás, como si hubiese visto al mismísimo duende de Salma ${ }^{4}$.

Les yeux de la jeune marocaine déclenchent le mouvement brusque du personnage qui se souvient d'abord du regard envoûtant de Salma, son amour de jeunesse puis de sa femme, Carlota, d'origine marocaine, décédée depuis dix ans. Dès les premières pages, la représentation du corps à travers l'enchâssement des regards, installe le lecteur dans la rencontre magique avec l'Autre et fonde la construction du récit entre rêve et réalité et dans le jeu discontinu de l'absent et du présent. Abel héberge ensuite, chez lui, Nadja qui lui apprend qu'elle est originaire de Tlata de Reixana, un petit village au nord-ouest du Maroc situé à peu de kilomètres du village de son enfance. Ses deux amis David Benasuly et Lidia Andrade l'aident à régulariser la situation de la jeune mère après avoir entendu le récit de sa découverte: "-Que son inmigrantes ilegales. Que han venido en patera. Que no tienen donde caerse muertos ... que están solos ... que no tienen familia $»^{5}$. Nadja raconte dans un espagnol approximatif comment les passeurs l'ont laissée sur la plage en lui disant qu'elle trouverait du travail en Espagne. Elle sera placée, pendant quelques mois, sous la tutelle d'Abel qui fera tout pour l'accepter ${ }^{6}$ et l'habituer à la ville, mais sans prévenir, elle repart un jour au Maroc avec le père de son enfant venu la rejoindre. Un retour mystérieux qui informe non seulement de la circulation migratoire autour de cette frontière mais aussi de la figure des nouveaux migrants, des jeunes femmes mineures, enceintes ou avec enfants qui prennent le risque de traverser, la nuit, les dangereuses vagues du Détroit de la Mer méditerranée considérée comme le cimetière de l'Europe. Le Détroit de Gibraltar, petit cordon ombilical de la Méditerranée, séparé de l'Espagne de seulement quatorze kilomètres est la voie de passage maritime la plus fréquentée par les migrants clandestins pour contourner, à l'ouest, le système de surveillance très perfectionné des deux enclaves espagnoles ${ }^{7}$, Ceuta, la plus proche du continent et Melilla plus à l'Est, unique frontière terrestre entre l'Afrique et l'Europe. Abel veut les retrouver et se rend d'abord à la Sous-préfecture de Málaga en charge du contrôle d'identité dans l'espace Schengen des immigrés clandestins désœuvrés et massés contre les abords des zones portuaires de la ville. Il se heurte au mur de plomb du vieil édifice où sont bloqués des migrants de toute nationalité :

Entró en el viejo edificio de paredes plomizas donde se ubica la Subdelegación del Gobierno.[...]A medida que subía los escalones, de escasa altura pero numerosos, Abel se vio obligado a detenerse[...]. La primera planta estaba atestada de gente. La 
mayoría eran marroquíes que se apiñaban en una de las puertas acristaladas, el resto se repartía entre negros africanos y algunos sudamericanos, todos ellos de diferentes países, sentados en silencio en los informales sillones negros de trazas modernistas y en los escalones que conducían a la segunda planta ${ }^{8}$.

Sans aucune trace de Nadja, Abel décide de sortir de Málaga pour se rendre au village de son enfance en voiture accompagné de son ami David. L'élaboration de la poétique des murs s'inscrit d'abord dans le déplacement du personnage qui franchit, sans obstacles, l'autre rive mais, à son arrivée, il est surpris par le dénuement du paysage qu'il découvre de l'autre côté du mur :

Veía a la mayoría de las mujeres con sus chilabas y sus jaiques y a los hombres con ropas gastadas y pasadas de moda. Los muros de la casa Raisuni estaban cubiertos de enredaderas y las plantas disimulaban las grietas inmisericordes y las manchas que los sucesivos inviernos se habían encargado de enhebrar en sus ladrillos9.

En effet, les fissures des murs qu'il observe sur la route reflètent la misère des classes sociales qui vivent aux abords des villes marocaines. À Larache, il est confronté ensuite aux murs de la vieille Medina où il recherche, péniblement, photo en main, dans les sinistres chambres qu'il visite, la jeune femme qui aurait été vue dans son village natal et avec sa famille. Partout, s'entassent devant lui, comme «des déchets et des âmes perdues ", des Sénégalais, des Mauritaniens, des Marocains et des exilés de l'Afrique subsaharienne :

Allí alrededor, en habitaciones abiertas, dormitaba una treintena de personas, en su mayoría de raza negra, junto a los que se reconocían también a los marroquíes más olvidados. Todos se hacinaban igual que desperdicios o que despojos, la escoria de la que nadie quería saber nada de nada; mejor muertos que ociosos, mucho mejor lejos, en países desarrollados que pudieran explotarlos a gusto que delinquiendo en su propia tierra. Formaban un buen cuadro de almas perdidas ${ }^{10}$.

Il croise, ensuite, près de la plage, d'autres migrants clandestins perdus, des survivants aux histoires multiples, sans doute victimes de passeurs sans scrupules, et qui errent chaque jour, dans l'attente d'un bateau pour atteindre l'Espagne. Un spectacle qui soulève en lui un sentiment mêlé de compassion et d'impuissance et qui lui fait mesurer le changement de la ville au $\mathrm{xxI}^{\mathrm{e}}$ siècle. Larache, située à plus de 100 kilomètres de la côte espagnole, est devenue la nouvelle plate-forme empruntée par les passeurs, plus à l'ouest, pour la traversée de la Méditerranée en deux jours, dans des embarcations de fortune, afin de contourner les nouveaux dispositifs de contrôle des forces de sécurité autour de la frontière. En effet, depuis la tragédie de $2005^{11}$ qui avait fait le tour du monde, une troisième barrière s'est érigée, et les murs relevés à plus de six mètres de hauteur puis renforcés par des kilomètres de clôtures de barbelés scellent hermétiquement la frontière à l'extérieur des points de contrôle réguliers. Selon Claude Quétel, les migrants «ne peuvent plus passer par cette voie ni par l'Atlantique où le flux migratoire clandestin a été jugulé par Frontex, l'agence européenne de protection des frontières de Schengen $»^{12}$. Aussi de nombreux clandestins refoulés de Ceuta qui n'ont pas réussi à escalader les grillages reculent et se réfugient à l'abri des caméras de surveillance, aux abords de Tanger, Nador, ou Tétouan où ils construisent eux-mêmes, des campements délabrés considérés comme " une troisième nation ou une salle d'attente $»^{13}$. À part Larache, ces petits refuges provisoires et invisibles, à l'extérieur de Ceuta et Melilla, contribuent à isoler ces deux enclaves des villes marocaines les plus proches et confrontent le personnage à la difficulté de traverser le mur du côté marocain. Le mur est à la fois un espace de circulation et de transit, de plus

Hispanismes, 18 | 2021 
en plus déplacé au $\mathrm{XxI}^{\mathrm{e}}$ siècle avec la mondialisation trépidante qui donne lieu à des récits et discours critiques sur l'Histoire entre l'Espagne et son ancienne colonie.

\section{Récits historiques et Discours critique sur les murs entre le Maroc et l'Espagne}

7 L'écriture bascule vers le passé et fait surgir d'autres ombres encore enfouies dans la mémoire du protagoniste quand le Maroc était alors territoire d'accueil pour de nombreux Espagnols, durant le Protectorat (1912-1956). Abel se souvient de Larache, premier lieu au Nord-ouest du Maroc du débarquement des troupes espagnoles en 1912, port de pêche et terre de ressources agricoles où vivaient ensemble, à son arrivée en 1931, avec les Marocains, des Espagnols et d'autres étrangers: Anglais, Français, Portugais et Juifs. Le narrateur convoque alors les souvenirs de son enfance qu'il redécouvre à travers les murs lézardés de l'ancienne maison de l'Espagne, pilier du pouvoir économique des familles espagnoles qui s'y étaient établis. Il retrouve, chez Samir, le guide marocain qui l'accueille à Larache, la même hospitalité et le même sentiment de solidarité qu'il avait connu auparavant, quand son père Abelardo, le chrétien, accueillait chez lui, avec les chansons de Noël, Mustapha Ben Laabi, le musulman et Jacobi Cohen, le juif, symbole de l'entente conviviale entre les trois cultures, un rappel de l'héritage culturel de l'Andalousie avant l'expulsion des Maures en1492. Le mur entre passé et présent s'abolit pour lui dans cette ville où s'affirme sa double identité rhizome de larachense et de malaguéen, sans barrière entre l'une et l'autre :

Vengo de Málaga, pero viví aquí muchos años. No sé si me creerá, pero siempre digo que soy de Larache. Aquí es donde fui realmente feliz. Cuando llegué, tenía siete años así que me crié en estas calles ${ }^{14}$.

8 La répétition $d u$ "je » est une réappropriation du territoire de son enfance, lieu d'identité et d'altérité où il a été heureux et d'où il fut brutalement arraché par les circonstances de l'Histoire. Son rapport à cette terre du Maroc est imprégné de ce passé et oriente l'écriture comme un parcours vers la quête de paternité. Abel Egea se souvient aussi du départ progressif des populations étrangères à la fin du Protectorat en 1956 jusqu'au départ définitif des Espagnols en 1975, avec la Marche Verte convoquée par le roi du Maroc, Hassan II qui avait incité son peuple à récupérer le Sahara occidental espagnol. Il raconte à Samir l'expérience personnelle encore douloureuse de l'expatriation de sa famille hors du Maroc, en 1971. Les points de suspension matérialisent le caractère fragmentaire de la mémoire du sujet qui retient la période vécue comme un double déracinement, ne se sentant pas reconnu par le Maroc ni par l'Espagne :

He vivido en un permanente desarraigo.[...] Los que nacieron en Marruecos y nada tenían que ver con el Ejército.[...]. Nosotros éramos felices en Larache. Muy felices... Pero llegó la marroquinización y no se nos permitió la doble nacionalidad, se nos exigía permiso de residencia. [...]

De pronto éramos extranjeros. Insensatos, creímos que, por consiguiente, éramos españoles... Allí,tampoco nos querían. Cuando llegamos a España, nos decían que íbamos a robarles los puestos de trabajo... Así de pronto no éramos españoles pero tampoco marroquíes...Y después de explicarte todo esto, ¿cómo explicarte que Larache es mi única bandera? Mis padres están enterrados aquí15. 
Cette intra histoire ${ }^{16}$ évoque, à travers le " je et le nous", l'exode de milliers d'Espagnols du Maroc, techniciens, et administratifs, arrivés en Espagne entre les années 1971 à 1976 et considérés comme une menace par leurs propres compatriotes. Au récit d'Abel, s'enchaîne celui de son ami David, originaire de Cádiz qui, après son exil en 1936 avec toute sa famille, fut, lui aussi, rapatrié à Madrid en 1975. David compare la situation de Nadja, jeune immigrée clandestine à la sienne quand il avait émigré à Tanger comme des milliers d'Andalous qui s'étaient réfugiés pendant la guerre civile au Maroc. L'articulation entre récit individuel et histoire collective plonge le lecteur au cœur du parcours migratoire des Espagnols qui a toujours existé vers le pays voisin depuis le $\mathrm{xxI}^{\mathrm{e}}$ siècle, pour des motifs politiques et économiques. Le récit laisse place ensuite au discours de David sur une autre période émouvante de l'Histoire, celle de l'émigration massive, vers le Maroc et vers l'Europe, de nombreux Espagnols fuyant le Franquisme et ses maux entre 1950 et 1973. La juxtaposition des deux voix enchâssées dans le roman confère à la narration du mur, des tonalités variées et dynamiques, mettant en lumière le paradoxe entre ceux qui sont refoulés du mauvais côté du mur et ceux qui sont du bon côté et qui continuent à venir s'installer encore à Larache au XxI ${ }^{\mathrm{e}}$ siècle depuis la crise de 2008. Le discours virulent de David résonne, dans le texte, comme un cri contre la mémoire courte des Espagnols, anciens émigrés au Maroc, qui oublient l'Histoire et considèrent la venue de l'immigré comme une perpétuelle menace de la perte de leur identité :

La puta gente de este país ha olvidado demasiado pronto que nosotros éramos los emigrantes que llenábamos los muelles de Argentina y de Marruecos, las estaciones de Alemania, los campos de refugiados de Francia... Eramos de cuarta, joder. Y ahora vamos por ahí con ese aire de gallitos prepotentes mirando los sudacas por encima del hombro y a los moros con el rabillo del ojo, desconfiando de ellos como si fuesen a cortarnos el cuello como los miserables cabrones que soltó Franco por los campos enemigos ${ }^{17}$.

Un discours critique qui fait allusion au traitement réservé aux Marocains que dénonce également le romancier espagnol Andrés Sorel dans son roman Las voces del Estrecho (2000) cité dans l'article d'Irene Andrés Suárez ${ }^{18}$. Le mur de la Méditerranée ne reflète plus seulement le contraste Nord-Sud. C'est un espace asymétrique, de plus en plus complexe qui acquiert une dimension politique et sociale et se définit, alors, dans le contexte de la mondialisation comme le lieu de tensions et de conflits permanents et de remises en question de négociations de projets culturels et économiques signés entre l'Espagne et le Maroc depuis 2000. Les contrats temporaires signés tous les ans entre l'Andalousie et le Maroc et les principes généraux du traité de Lisbonne de bon voisinage et de coopération signé en 2007 sont autant de faits politiques qui interrogent l'existence du mur et des barrières ${ }^{19}$ entre les deux pays. Des procédures qui font écho aux propos de Glissant et Chamoiseau dans l'essai publié en 2007, Quand les murs tombent: " Ce n'est pas l'immigration qui menace ou appauvrit, c'est la raideur du mur et la clôture de soi $»^{20}$. Abel dénonce la vacuité des frontières et l'européanisation des clôtures de Ceuta et Melilla qui perpétue l'esprit colonial opposant les dominants aux dominés :

Fue en sa misma habitación, donde Abel comprendió la vacuidad de nuestras fronteras, la artificiosidad de nuestras diferencias, esa impostura instalada en el poder que nos conduce a ciegas a seguir a unos líderes, manipuladores y ególatras ${ }^{21}$.

11 Un discours humaniste qui englobe dans le présent, le passé et le futur et interpelle le lecteur sur le sens de la frontière commune entre les deux pays tout au long de 
l'Histoire. Abel exhorte les Espagnols à réfléchir sur le sort futur de leurs petits enfants qui pourraient un jour quitter involontairement l'Espagne à leur tour :

Todos somos emigrantes. Créame. Ahora gozamos de una buena racha, pero nadie puede asegurarnos que dentro de cuarenta años, no estarán nuestros nietos cruzando el mar para buscarse su futuro. El mundo es redondo y da muchas vueltas $^{22}$.

12 Le croisement des pronoms personnels «je/nous» dans la phrase Nous sommes tous des émigrés, révèle son souhait d'abolir les tiroirs identitaires au profit d'une humanité plurielle qui défend la valeur universelle de la perception de l'Autre. Les différentes perspectives temporelles qui fragmentent le roman, la superposition des récits et l'imbrication des discours subversifs l'un dans l'autre, sont autant de résonances de la complexité du mur, à la fois dans ses ruptures et dans la continuité de ses constructions imaginaires et politiques au fil de l'Histoire. Des modalités d'écritures du mur qui contiennent des messages politiques et qui corroborent la définition que donne l'historien Claude Quétel de la fonction du mur : Ce sont des constructions qui font de la politique, qui disent quelque chose $\aleph^{23}$. La vision politique du romancier informe de la difficile gestion pour l'Espagne du Détroit ${ }^{24}$, d'un côté, le désespoir des immigrants bloqués au Maroc et de l'autre, la logique sécuritaire de l'Europe forteresse et ses mesures de protection contre l'immigration, le terrorisme, et les trafics illicites de drogue et d'êtres humains. Tous ces facteurs contribuent au xxI siècle à rendre compte des discussions controversées qui, en permanence, bouleversent le sens de ce mur du Sud qui ne finit pas de déborder, de s'allonger avec l'arrivée massive de migrants à la merci de passeurs trafiquants qui inventent de nouvelles stratégies migratoires au rythme de l'avancée des réseaux sociaux.

13 Au terme de son séjour à Larache, il croise sur la route, Nadja heureuse en compagnie du père de son enfant, mais ne s'arrête pas, et au fur et à mesure qu'il les perdait de vue dans le rétroviseur, leurs silhouettes s'y reflétaient comme des ombres pâlies. Une scène qui illustre les deux côtés du mur, à la fois, la réalité de l'échec des projets des jeunes migrants marocains qui n'ont pas réussi à franchir le mur de l'Europe et l'imaginaire du déplacement, avec la mise en abyme ou la petite fenêtre ouverte sur Nadja, sorte de pont vers les anciennes ombres du protagoniste de retour à Málaga :

Cuando cruzó el puente que salva el río Lükus, el corazón le dio un repentino vuelco [...] al borde de la carretera, una chica caminaba con un niño a la cintura [...] a su lado, con una cesta de mimbre en la mano, un hombre alto [...]. Los dos reían. [...] A medida que los perdía en el espejo retrovisor, la carretera se fue oscuriendo, hasta que Abel Egea no tuvo más remedio que encender las luces cortas[...].Trató de descubrirlos forzando la vista, pero en el espejo ya sólo se reflejaba un escenario de sombras enmustiadas ${ }^{25}$.

Les allers et retours entre les deux rives du Détroit constituent le trait d'union entre les trois personnages malgré l'échec de Nadja et enrichissent la fonction du mur dans son rapport au temps comme le chronotope bakhtinien: espace discursif, points de vue politiques, lieu de rencontres avec d'autres langages et cultures diverses, lieu de mémoire historique, lieu de mémoire familiale et d'expériences affectives vécues qui imprègnent la poétique de l'entre-deux et met en lumière le rôle dynamique du mur dans la relation avec l'Autre. 


\section{De la relation avec Nadja}

15 Le mouvement du personnage principal est en effet une expérience temporelle. Il aspire comme tout déraciné à revenir à son point de départ. Abel, nom d'origine biblique, incarne aussi la figure de l'homme pieux et juste dont la condition itinérante de pasteur nomade implique la spiritualité et la relation à l'Autre. L'écriture du mur est indissociable de l'imaginaire de la quête intime de soi inscrite dans la charge poétique des sibilantes du titre: Sombras en sepia. Le personnage, double de l'auteur, retrouve une certaine sérénité en plongeant dans le monde de son enfance méditerranéenne. Les premières ombres successives qui surgissent dans sa mémoire, sont celles de trois figures avec leurs valises, dans un couloir, avec son père, sa mère Nuria, portant dans ses bras, sa sœur, Lucia, et lui, à l'âge de sept ans, arrivant pour la première fois à Larache. Le souvenir de ce moment intime et nostalgique de l'enfance est enchâssé telle une mise en abyme dans l'image d'une photo en noir et blanc, jaunie avec le temps, couleur sépia qu'Abel a gardée depuis cinquante ans. Il s'agit du souvenir de Salma, un amour de jeunesse, ravivé par la puissance du regard perçant de Nadja :

Su forma de hablar seguía atrapando a Abel. Al escucharla le invadía un sentimiento de amparo,de refugio, de cariño. Era como si ahora supiese cuál era el sentido de cada día. [...] Volvió a observarla con entusiasmo. Había algo en Nadja que a Abel le parecía distinto, embaucador, irremediablemente atrayente. Se confundían en su cabeza el acento cándido de sus palabras en español con los movimientos de sus brazos y de la cabeza, herederos del más ancestral arte de la seducción, [...] con el embrujo de sus ojos aceitunos [...] con la delicadeza de sus largos dedos y con la ineludible tentación de sus labios amaestrados ${ }^{26}$.

Les cinq sens élémentaires (goût, odorat, toucher, vue, ouïe) sont convoqués pour décrire le désir et l'émotion d'Abel fasciné par le charme oriental du corps et l'expression gestuelle de Nadja. Il garde en mémoire les odeurs de thé, de fleur d'oranger, des épices des petits plats d'agneau qu'elle lui préparait à Málaga pendant la brève période de son séjour chez lui. Des saveurs de son enfance qui stimulent son imagination et éveillent en lui des désirs passionnés de sa jeunesse. Elles constituent aussi la part dynamique de son identité inscrite dans la relation à l'Autre décrite par Glissant dans Poétique de la Relation: "La relation à l'Autre (à tout l'Autre, dans ses présences animales, végétales et culturelles et par conséquent humaines) nous indique la part la plus haute, la plus honorable, la plus enrichissante de nous-mêmes. Que tombent les murs $»^{27}$.

17 Au contact de Nadja, son corps remobilisé, s'anime et devient le lieu des rêveries érotiques qu'il avait connues avec Salma et Carlota. Il se sent l'homme le plus heureux au monde et rêve de fonder une famille. Il rêve qu'ils sont nus tous deux au lit et les frissons qu'il ressent de la sensualité des doigts de la jeune fille, réveillent les souvenirs endormis de son corps au point de lui faire revivre les secrets de ses premiers émois sexuels :

Notó también cómo se decubría su manta y que Nadja se deslizaba junto a él. [...]. Pero ella se apretó contra él y lo abrazó por la espalda. La boca se le secó como si llevara días sin beber una gota. [...] ¿Qué estás haciendo? balbuceó al fin. Pero su pregunta carecía de autoridad. [...] Finalmente, se dejó hacer. La chica lo desvistió entre las sábanas mojadas cuando hubo acabado, volvió a pegar su suave cuerpo al de Abel. El sintió unos senos erectos en su espalda ${ }^{28}$.

Le mur intérieur du personnage se fissure pour délivrer cette part primitive, opaque, qui lui fait oublier la monotonie de son existence et surtout la solitude du présent. 
L'imagination érotique donne lieu à une écriture du corps qui exsude toutes ses humeurs illustrant l'intensité des émotions du sujet, que l'écrivain communique au lecteur comme la passion décrite par Roland Barthes dans Poétique du récit qui est " celle du sens, c'est-à-dire d'un ordre supérieur de la relation, qui possède, lui aussi, ses émotions, ses espoirs, ses menaces, ses triomphes $»^{29}$. Le narrateur révèle comment Abel assailli par le désir, transgresse les barrières de son âge et sort, pendant un moment, des "murs d'une solitude forcée »mais cette étreinte érotique est interrompue brusquement par le réveil du petit Zacarías :

Un impulso natural, masculino, lo lanzaba sin demora sobre el cuerpo apetecible de la chica. [...]. Sentía los dedos de Nadja asiendo con impaciencia toda su masculinidad, con una emergencia que sólo reconocen los que viven entre recuerdos o entre las paredes de una soledad obligada. Abel no era capaz de darse la vuelta [...]. Su boca sedienta de la boca de Nadja, sólo fue capaz de masticar un remordimiento acuciante [...] cuando Zacarías rompió a llorar en el otro dormitorio ${ }^{30}$.

19 Le réveil de l'enfant interrompt la transgression du désir sexuel qui suppose la ligne symbolique à ne pas franchir, obligeant Abel à sortir brutalement du lit et à se réfugier dans la salle de bain. La confrontation avec son double dans le miroir est une autre mise en scène de la perception des limites de son corps réel ; il s'interroge tout en observant les moustaches blanchesqui le ramènent à la raison : il ne peut pas faire l'amour avec une jeune fille de dix-sept ans :
Abel había dado un salto en la cama y se refugió en el cuarto de baño [...]. La luz del baño lo deslumbró unos segundos y luego se vio reflejado en el espejo sin reconocerse. Tenía enfrente un hombre de piel blancuza, de hombros caídos, [...] el pequeño bigote salteado de canas y la papada cada día más proeminente sólo acentuaban la sensación de estar cruzando una difusa frontera tras la que temía encontrarse de un momento a otro. [...]. No era capaz de hacer el amor con Nadja. Todo se reducía a eso ${ }^{31}$.

20 Le personnage, sans doute par pudeur, réfrène ses pulsions et accepte ses limites: «Abel añoró su juventud y aceptó el hecho de que jamás podría ser el hombre que ella necesitaba ni el que, pobablemente, había estado soñando $»^{32}$. L'image de l'effondrement des murs de la maison sur sa tête, l'obscurité et l'image du recueillement dans le mausolée, constituent l'une des visions les plus poétiques de la fin de la rêverie érotique du personnage. L'ombre grise qui l'enveloppe et l'odeur de la naphtaline illustrent les profondeurs de l'être encore englouti dans son passé et confronté à ses absences :

Las paredes de la casa se inclinaron sobre su cabeza, como si el techo se viniese abajo muy lentamente, y la cristalera se oscureció con una sombra siniestra y gris.Toda la casa se transformó en un máusoleo que olía a naftalina. Abel pensó en marcharse, pero se limitó a sentarse en el sillón ${ }^{33}$.

21 L'articulation de l'intérieur et l'extérieur du corps fait écho aux deux côtés du mur, sa part d'invisible et de visible. L'expérience bouleversante d'Abel est l'épreuve de tout être humain qui rencontre l'altérité à travers le rêve d'une belle histoire d'amour. L'insistance sur cet état vague et diffus où se loge l'ombre à l'intérieur du corps de l'homme nous confronte tous à nos propres limites, à nos silences intérieurs, à notre manière de transgresser l'interdit et de gérer nos distances avec l'Autre. Cette expérience intérieure et imaginaire du sujet ouvre des perspectives encore plus larges pour aborder la définition du mur. Ne serait-elle pas identique à celle des migrants 
bloqués ou dans l'attente, qui, devant le mur infranchissable, rêvent aussi de s'épanouir et d'un ailleurs paradisiaque ${ }^{34}$ loin de leur espace d'origine? inséparables à travers le corps et le mouvement circulaire du personnage combinant vision politique et dimension poétique. Toute l'aventure d'Abel révèle combien le mur est un sujet infini et fécond dans la littérature, territoire absolu de liberté qui nourrit l'imaginaire et offre la possibilité d'interroger le monde et se recomposer avec l'Autre. Sergio Barce, écrivain nomade, fait, à l'instar de Blanchot, le saut ${ }^{40}$ de l'autre côté vers un espace ouvert par le mouvement d'écrire, un voyage qui abolit le mur, et qui l'ouvre vers d'autres rêves, d'autres lignes d'écriture qui fécondent tout l'univers fictionnel de ses romans écrits depuis 2003. Il obtient en 2006 le Prix Murcia des trois cultures. Jean 
Genêt (1910-1986), écrivain et poète français et Juan Goytisolo ${ }^{41}$ (1931-2017), romancier espagnol de la Génération 50 sont enterrés à Larache.

\section{BIBLIOGRAPHIE}

Irene ANDRES SUÁREZ (2004), «Inmigración y Literatura española actual : Las Voces del Estrecho » [on-line]. Revista de literatura y cultura, Neufchâtel, p. 7-24 [consulté le 8 mars 2021] <URL:https://www.kb.osu.edu/dspace/bitstream/handle/1811/77517/EC_V17N1_007.pdf>.

Mikhaïl BAKHTINE, Esthétique et théorie du roman, Paris, Gallimard, 1978.

Roland BARTHES, Le degré zéro de l'écriture, Paris, Seuil, 1972.

Roland BARTHEs, Poétique du récit, Paris, Seuil, 1977.

Maurice BLANCHOT, L'espace littéraire, Paris, Gallimard, 1955.

Patrick CHAMOISEAU, Frères migrants, Paris, Seuil, 2017.

Mircea ELIADE, Le mythe de l'Eternel retour, Paris, Gallimard, 1969.

Sergio BARCE GALLARDO, Sombras en sepia, Valencia, Pre-Textos, 2006.

Elsa GANIVET, Esthétique du mur géopolitique, Québec, Presses universitaires du Québec, 2015.

Édouard GLISSANT, L'intention poétique, Paris, Seuil, 1969.

Édouard GLISSANT, Poétique de la Relation, Paris, Gallimard, 1990.

Édouard GLISSANT et Patrick CHAMOISEAU, Quand les murs tombent : l'Identité nationale hors la loi ?, Paris, Galaade, 2007.

Norbert HILAIRE, L'Expérience esthétique des lieux, Paris, L'Harmattan, 2008.

Mohamed KHACHANI, La emigración subsahariana:Marruecos como espacio de tránsito, Revue Migraciones, n 10, Fundació CIDOB, Barcelona, 2006.

Khadija KARZAZI, « La inmigación marroquí en la narrativa española contemporánea », F. J. García Castaño (coord.), Actos del Congreso internacional sobre Migraciones en Andalucía, Migraciones, Granada, Instituto de Migraciones, 2011, p.287-293.

Pierre MINICONI, « Unamuno, entre histoire et littérature », Acta fabula, vol. 6, nº 2 (2005), [consulté le 8 mars 2021] <URL : http://www.fabula.org/revue/document918.php>.

Alberto MONTEAGUDO CANALES, « Pateras Z: La zombificación de la inmigración norteafricana en Las Voces del Estrecho », LL Journal, vol. 13, n² 2, 2018, p. 49-64, Pdf consulté le 10 mars 2021.

Georges PÉREC, Espèces d'espaces, Paris, Éditions Galilée, 1974.

Claude QUÉTEL, « Murs contre l'immigration illégale », Histoire des murs. Une autre histoire des hommes, Paris, Perrin, col. Tempus, 2012, [consulté en mars 2021] <URL : https://www.cairn.info/ histoire-des-murs--9782262039547-page-243.htm>. 
Claude QUÉTEL, (oct. 2015) « Les murs font de la politique ». Migrants, la politique des barbelés, (entretien), $\mathrm{n}^{\circ} 77$, hebdo [consulté en mars 2021] < URL : https://le1hebdo.fr/journal/migrantsla-politique-des-barbeles/77/article/les-murs-font-de-la-politique-1228.html>.

Saïd SADDIKI (mars 2012), « Les clôtures de Ceuta et de Melilla. Une frontière européenne multidimensionnelle », revue Études internationales, vol. 43, nº 1, Éditeur(s) Institut québécois des hautes études internationales, [consulté en mars 2021] <URL : https://www.erudit.org/fr/ revues/ei/2012-v43-n1-ei5009237/1009139ar.pdf>.

\section{NOTES}

1. Sergio BARCE GALLARDo, Sombras en sepia, Valencia, Pre-Textos, 2006.

2. «temps-espace »: la corrélation essentielle des rapports spatio-temporels, telle qu'elle a été assimilée par la littérature. Mikhaïl BAKHTINE, Esthétique et théorie du roman, Paris, Gallimard, 1978, p. 237.

3. «Poétique des profondeurs. [...]. Extension vertigineuse, non pas sur le monde, mais vers les abîmes que l'homme porte en lui », Édouard GLISSANT, Poétique de la Relation, Gallimard, 1990, p. 36.

4. Sergio BARCE GALLARDO, op. cit., p. 10.

5. Ibid., p. 31.

6. «Accepter l'opacité de l'Autre, tout l'Autre, l'imprévisibilité de ses choix, de sa nature réelle, c'est déjà renoncer à dominer l'Autre, à dominer tout autre et par là à dominer le monde ". Patrick CHAMOISEAU, Frères migrants, Paris, Seuil, 2017, p. 88.

7. Ceuta et Melilla sont sous contrôle espagnol, Melilla depuis 1497 et Ceuta depuis 1668.

8. Sergio baRCE GALLARDo, op. cit., p. 89-90.

9. Ibid., p. 39.

10. Ibid., p. 131-132.

11. En 2005, cinq clandestins sont morts et plusieurs ont été blessés après avoir franchi illégalement la barrière de l'enclave espagnole de Ceuta. Cf. «L'assaut d'immigrants sur l'enclave espagnole de Ceuta a fait cinq morts", Le Monde (29/05/2005) [consulté le 3 mars 2021] <URL : https://www.lemonde.fr/international/article/2005/09/29/1-assaut-d-immigrants-sur-l-enclaveespagnole-de-ceuta-a-fait-cinq-morts_694052_3210.html>.

12. «Après avoir condamné le mur de Bush, l'Union européenne a financé dix ans plus tard le premier mur contre l'immigration illégale à Ceuta et Melilla, deux enclaves espagnoles situées au nord du Maroc. C'est le premier mur de l'espace Schengen, des barrières métalliques d'autant plus efficaces que l'espace est très restreint. Frontex est le bras armé de l'UE sur les frontières extérieures de l'espace Schengen ». Entretien avec Claude QUÉTEL, "Les murs font de la politique », Question 4, Octobre 2015 < URL : https://le1hebdo.fr/journal/migrants-la-politiquedes-barbeles/77/article/les-murs-font-de-la-politique-1228.html>

13. «Un grand nombre d'immigrants irréguliers qui n'ont pas réussi à entrer en Europe ou ne veulent pas courir le risque d'y aller construisent sur le territoire marocain, près de Ceuta et de Melilla, des implantations provisoires considérées comme « une troisième nation » ou « une salle d'attente ", où ils ne peuvent ni atteindre leur Eldorado, ni retourner dans leurs pays d'origine ». Saïd SADIKKI, «Les clôtures de Ceuta et de Melilla. Une frontière européenne multidimensionnelle ", revue Études internationales [on-line], Éd. Institut québécois des hautes études internationales, vol. 43, no 1 (2012), p. 49-65 [consulté le 3 mars 2021] <URL : https:// www.erudit.org/fr/revues/ei/2012-v43-n1-ei5009237/1009139ar.pdf>.

14. Sergio BARCE GALLARDo, op. cit., p. 49.

15. Ibid., p. 56. 
16. L'« intra histoire » que l'on peut définir comme ce qui fait l'essence du vécu de ses acteurs à l'intérieur de ce que Ortega y Gasset appelait leur « circunstancia ». Pierre MINICoNI, « Unamuno, entre histoire et littérature », Acta fabula, vol. 6, n 2 (2005), [consulté le 8 mars 2021] <URL : https://www.fabula.org/acta/document918.php>.

17. Sergio BARCE GALLARDO, op. cit., p. 116.

18. «El escritor nos recuerda que el desarrollo económico actual ha vuelto a los españoles arrogantes e insensibles a los problemas de esos inmigrantes que llegan en busca de un mínimo de bienestar, también les ha hecho olvidar que hace apenas unas décadas muchos de los nuestros se vieron obligados a emprender el éxodo por los mismos motivos", Irene ANDRES SUÁREZ, «Inmigración y Literatura española actual : Las Voces del Estrecho » [on-line]. Revista de literatura y cultura, Neufchâtel, 2004, p. 19 [consulté le 8 mars 2021] <URL: https://kb.osu.edu/handle/ 1811/77517?show=full>.

19. «Depuis deux décennies, la Méditerranée regorge d'une multitude de projets de coopération culturelle et économique, mais de nouveaux murs matériels et virtuels, destinés à réaliser « la forteresse Europe ", s'y sont également édifiés ». Said SADIKKI, op. cit., p. 1.

20. Édouard GLISSANT et Patrick CHAMOISEAU, Quand les murs tombent : l'Identité nationale hors la loi ?, Paris, Galaade, 2007, p. 24.

21. Sergio BARCE GALLARDO, op. cit ., p. 136.

22. Ibid., p. 98.

23. Claude QUÉTEL (oct.2015), «Les murs font de la politique ». Migrants, la politique des barbelés, $\mathrm{n}^{\circ} 77$ [consulté en mars 2021] < URL: https://le1hebdo.fr/journal/migrants-la-politique-desbarbeles/77/article/les-murs-font-de-la-politique-1228.html>.

24. «El caso de los acuerdos firmados entre España y Marruecos en materia pesquera: un sector que, al combinar los recursos naturales con las fronteras marítimas nacionales, ha constituido tradicionalmente un capítulo de especial relevancia en las relaciones entre ambos países desde la independencia marroquí del Protectorado español. Aunque no faltos de retos, estos tratados siempre habían sido de naturaleza bilateral, beneficiando a ambas partes. Sin embargo, la admisión de España en la Comunidad Económica Europea rompió el equilibrio de poder que existía ». Alberto MONTEAGUdo CANALES, «Pateras Z: La zombificación de la inmigración norteafricana en Las Voces del Estrecho », LL Journal, New York, vol. 13, nº 2 (2018), p. 5.

25. Sergio BARCE GALLARDO, op. cit., p. 200-201.

26. Ibid., p. 183-184.

27. Édouard GLISSANT, Patrick CHAMOISEAU, op. cit., p. 25.

28. Sergio BARCE GALLARDO, op. cit., p. 187-188.

29. Roland BARTHES, Poétique du récit, Paris, Seuil, 1977, p. 52.

30. Sergio BARCE GALLARDO, op. cit., p. 189-190.

31. Ibid., p. 190.

32. Ibid., p. 216.

33. Id.

34. «Las voces que llenan este libro [...] hablan de unos personajes que se han extraviado por el camino en su búsqueda del Paraíso, sorprendidos por espejismos sórdidos y caprichosos ", Andrés SUÁREZ, op. cit., p. 22.

35. Édouard GLISSANT, L'intention poétique, Paris, Seuil, 1969, p. 182.

36. Roland BARTHES, Le degré zéro de l'écriture, Paris, Seuil, 1972, p. 17.

37. Sergio BARCE GALLARDO, op. cit., p. 240.

38. Mircea ELIADE, Le mythe de l'Eternel retour, Paris, Gallimard, 1969, p. 85-86.

39. Édouard GLISSANT, Poétique de la Relation, Paris, Gallimard, 1990, p. 197.

40. «Le saut est la forme ou le mouvement de l'inspiration », Maurice BLANCHOT, L'espace littéraire, Paris, Gallimard, 1955, p. 233. 
41. Son roman España y sus ejidos (2003) aborde le thème de l'émigration en Andalousie et dénonce les conditions de vie infrahumaines des travailleurs Marocains dans les serres de El Ejido au Sud de l'Espagne.

\section{RÉSUMÉS}

Sombras en sepia raconte l'aventure d'un vieil homme, Abel, qui découvre sur une plage andalouse, une jeune marocaine Nadja, accompagnée de son fils. Cette rencontre bouleversante lui rappelle les souvenirs les plus intimes de sa jeunesse à Larache, un village du nord-ouest du Maroc qu'il décide de revisiter avec un ami. Le déplacement de Málaga à Larache interroge autant la complexité des récentes barrières construites à la frontière sud pour freiner les migrants que les relations tissées entre l'Espagne et son ancien protectorat depuis 1912. Dans le voyage circulaire du protagoniste, le mur s'annule dans la Relation à l'Autre, entre réel et rêve, nomadisme et migration, les deux faces de l'univers esthétique des romans de Sergio Barce Gallardo écrits depuis 2003.

Sombras en Sepia narrates the adventures of an old man, Abel, starting on an Andalusian beach where he discovers Nadja, a young Moroccan girl with her son. This encounter reminds him of many intimate childhood memories in Larache, in the northwestern part of Morocco, and motivates him to revisit with a friend this city. His move from Málaga to Larache questions both the complexity of the barriers recently built on the southern Spanish border to curb migration, as well as the evolution of the relationship between Spain and her former colony since 1912. In this round trip in which the protagonist uncovers, the border slowly fades between reality and dream, nomadism and migration, the two facets of the aesthetic universe created by Sergio Barce Gallardo in his novels since 2003.

\section{INDEX}

Keywords : 21st century, novel, Spain, migration, border, identity, alterity

Mots-clés : roman, xxie siècle, Espagne, migration, mur, identité, altérité

\section{AUTEUR}

\section{LYDIE ROYER}

Université de Reims Champagne Ardennes 